\title{
PAX6 expression in the developing human eye
} Sachiko Nishina, Shinichi Kohsaka, Yuki Yamaguchi, Hiroshi Handa, Atsushi Kawakami,
Hajime Fujisawa, Noriyuki Azuma

\begin{abstract}
Aims-To investigate the changes in PAX6 expression in the developing human eye. Methods-Six developing human eyes from 6 to 22 weeks' gestation were evaluated. Frozen sections were immunohistochemically stained with monoclonal antibody to chick Pax6 (amino acids 1-223). To verify antibody specificity, western blot analysis was carried out using cell lysates from P19 cells transfected with the human $P A X 6$ gene.

Results-Western blot analysis demonstrated that the antibody reacted to human PAX6 protein. Positive immunostainings for PAX6 were seen in the surface ectoderm, lens vesicle, inner and outer layers of the optic cup, and optic stalk at 6 weeks, and in the corneal epithelia and conjunctiva, lens, and nonpigmented ciliary epithelia from 8 to 22 weeks. In the retina, positive cells were seen in the entire retina from 8 to 10 weeks, and were restricted to the ganglion cell layer and the inner and outer portions of the inner nuclear layer after 21 weeks. Conclusions-PAX6 is expressed on the surface and neuroectoderms at an early stage, then in the differentiating cells in the cornea, lens, ciliary body, and retina through development. PAX6 may play a role in determining cell fate in the morphogenesis of various human ocular tissue. (Br F Ophthalmol 1999;83:723-727)
\end{abstract}

Bepartment of Engineering, Faculty of Bioscience and Biotechnology, Tokyo Institute of Technology,

Kanagawa, Japan

Y Yamaguchi

H Handa

Division of Biological Science, Nagoya

University Graduate

School of Science,

Nagoya, Japan

A Kawakami

H Fujisawa

Correspondence to: Noriyuki Azuma, MD, Department of

Ophthalmology, National

Children's Hospital, 3-35-31

Taishido, Setagaya-ku,

Tokyo, 154-8509, Japan.

Accepted for publication 11 January 1999 expression, so it has been suggested to be master control gene for morphogenesis of compound eyes in Drosophila melanogaster. ${ }^{1}$ Recent studies have shown that three other Drosophila genes, sine oculis (so), eyes absent (eya), and dachshund (dac), also can result in the development of ectopic eyes, and have suggested that these genes may act downstream of ey or form a regulatory network during multiple steps of ocular development in Drosophila. ${ }^{2-4}$ Pax6 is widely present and highly conserved in various vertebrate and inverte- brate species, and the gene is thought to play the most critical role in ocular formation in animals. ${ }^{4}$

A previously reported investigation ${ }^{5}$ of murine Pax6 showed that the gene is expressed in the developing central nervous system, eye, pituitary, and nasal epithelium. In the eye, it is first detected in the optic sulcus, then in the optic vesicle, surface and neuroectoderms, and strongly in the inner layer of the cup, the lens, and the cornea. ${ }^{5}$ Pax6 expression also was investigated in many other species by in situ hybridisation or immunohistochemistry. Recent investigations in developing goldfish, ${ }^{6}$ Xenopus, ${ }^{7}$ mice, ${ }^{8}$ and chicks ${ }^{9}$ showed its uniform pattern of expression in the entire retina in the early developmental phase; the expression then was restricted to the mitotically active inner layer cells in the late phase. These results indicate a relation between Pax6 expression and retinal cell differentiation.

A cellular mechanism activated by PAX6 has not been determined, but several reports implicated possible target genes such as the neural cell adhesion molecule (N-CAM), ${ }^{10}$ crystalline, ${ }^{11}$ R-cadherin, ${ }^{12}$ and rhodopsin $1 .{ }^{13}$ PAX6 may regulate various tissue specific genes, and be involved in induction, mitosis, regeneration, and differentiation during development.

Mutations in the PAX6 gene and a variety of phenotypes that range from the anterior segment to the fundus, also indicate multiple functions of the gene. Mutations in the PAX6 gene cause aniridia, ${ }^{14}$ Peters' anomaly, ${ }^{15}$ congenital cataracts, anophthalmia, ${ }^{16}$ autosomal dominant keratitis, ${ }^{17}$ and foveal hypoplasia. ${ }^{18}$ However, few reports have described PAX6 expression in the human embryo, and their data were limited to the early stages of development, 3-7 weeks' gestation. ${ }^{14}{ }^{19}$ To clarify the influence of PAX6 on human ocular development and malformations, we investigated PAX6 expression in developing human eyes by immunohistochemistry.

\section{Materials and methods}

\section{SPECIMENS}

The present study was conducted in accordance with the World Medical Association Declaration of Helsinki. The use of embryonic and fetal tissues was approved by the National Children's Hospital experimental review board and deemed exempt from regulations applicable to human subjects. Six developing human 


\section{A PAX6 plasmid}

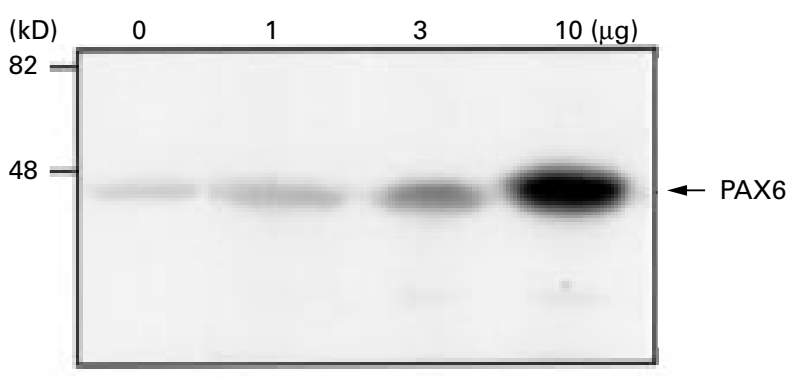

B PAX6-5a plasmid

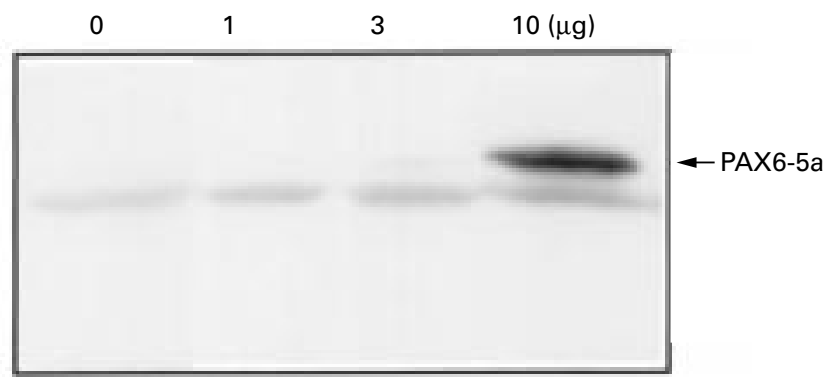

Figure 1 Western blot analysis of P19 cells transfected with plasmids expressing PAX6 (A) or PAX6-5a (B). The amounts of plasmids were 0, 1 , 3, and $10 \mu \mathrm{g}$. The anti-chick Pax6 antibody detects bands at the molecular weights of 46 and $48 \mathrm{kDa}$ in a dose dependent manner.

eyes at $6,8,9,10,21$, and 22 weeks' gestation were obtained after spontaneous or legal abortions. The eyes were obtained from fetuses that had no systemic or ocular anomalies. The ocular tissue from each fetus appeared normal upon microscopic examination.

MONOCLONAL ANTIBODY TO PAX6

The DNA region corresponding to amino acids 1-223 of chick Pax6 was cloned by polymerase chain reaction into the Escherichia coli expression vector. Recombinant protein was expressed and purified. The monoclonal antibody was generated by immunising mice with the recombinant protein as described previously. ${ }^{9}$

\section{WESTERN BLOT ANALYSIS}

Mouse embryonal carcinoma P19 cells were transfected with various amounts of plasmids $(0,1,3$, and $10 \mu \mathrm{g})$ expressing human PAX6 or PAX6-5a protein as described previously. ${ }^{20}$ PAX6-5a has an additional 14 amino acid insertion in the paired domain that is encoded by exon 5a of a splice variant. ${ }^{21}{ }^{22}$ After 40 hours, cells were lysed with $50 \mathrm{mM}$ TRIS- $\mathrm{HCl}$ (pH 7.9)/500 mM KCl/1\% NP-40. The whole cell lysates $(100 \mu \mathrm{g})$ were subjected to $10 \%$ SDS-PAGE, and transferred onto polyvinylidene difluoride membranes. Blots were sequentially incubated with anti-chick Pax6 monoclonal antibody ${ }^{9}$ (1:1000 dilution), biotinylated anti-mouse IgG secondary antibody, and streptavidin conjugated horseradish peroxidase, and visualised using the ECL system (Amersham, Bucks) as described by the manufacturer.

\section{IMMUNOHISTOCHEMISTRY}

Specimens were fixed in $4 \%$ paraformaldehyde in $100 \mathrm{mM}$ phosphate buffer for 3 hours, and embedded and frozen in Tissu Mount (Chiba Medical Co, Saitama, Japan). Each block was serially sectioned at $5-10 \mu \mathrm{m}$ on a cryocut (Reichert-Jung). Sections first were immersed in $6 \%$ hydrogen peroxide in methanol for 30 minutes to counteract endogenous peroxidase and washed with TBS (10 mM TRIS-HCl, $130 \mathrm{mM} \mathrm{NaCl}, \mathrm{pH}$ 7.4). They then were blocked with 5\% skim milk dissolved in TBS for 30 minutes. PAX6 antibody was diluted to a ratio of 1:1000 with TBS, applied to each section, and stored overnight at $4^{\circ} \mathrm{C}$ in a moist chamber. Sections then were washed with TBS and incubated with biotinylated anti-mouse secondary antibody diluted 1:400 (Amersham) for 1 hour at room temperature. Sections were washed with TBS and incubated with $\mathrm{ABC}$ reagent (Vectastain, Burlingame, CA, USA) for 30 minutes at room temperature. Sections were again washed with TBS. Peroxidase activity was seen after incubation in TBS containing $0.03 \%$ hydrogen peroxide and $0.05 \% \mathrm{w} / \mathrm{v}$ diaminobenzidine tetrahydrochloride (DAB). Each section also was immunostained using alkaline phosphatase and a substrate system of 5-bromo-4-chloro-3-indoxyl phosphate and nitro blue tetrazolium chloride (BCIP/NBT) (Dako, Carpinteria, CA, USA). Sections were rinsed with TBS and mounted without counterstaining.

\section{Results}

WESTERN BLOT ANALYSIS

The antibody recognised human $46 \mathrm{kDa}$ PAX6 (Fig 1(A), 1, 3, $10 \mu \mathrm{g}$ ) and $48 \mathrm{kDa}$ PAX6-5a isoforms (Fig 1(B), 1, 3, $10 \mu \mathrm{g}$ ) in a dose dependent manner. The control $(0 \mu \mathrm{g})$ showed a weak band, which probably was a reaction to endogenous Pax6 in P19 cells. These results clearly showed that the monoclonal anti-Pax6 antibody used in this study was specific for both PAX6 and PAX6-5a splice variants with molecular weights of 46 and $48 \mathrm{kDa}$.

IMMUNOHISTOCHEMISTRY

Positive immunostaining for the PAX6 protein was seen in cell nuclei of the surface ectoderm, lens vesicle, the inner and outer layers of the optic cup, and faintly in the optic stalk at 6

Figure 2 (A) Photomicrograph of developing human eye immunostained with PAX6 antibody at 6 weeks. Positive immunostaining is seen on the surface ectoderm, lens, optic cup, and optic stalk. (B) Photomicrograph of developing human eye immunostained with PAX6 antibody at 10 weeks. Positive staining is seen in the epithelia of the cornea, conjunctiva, and lens, the primordia of the iris and ciliary epithelia, and the inner and outer neuroblastic retinal layers. (C) Magnified view of the anterior segment at 10 weeks. Con = conjunctiva; $C=$ cornea. (D) The cornea at 21 weeks. Positive staining is seen in the epithelium (arrowheads). (E) The anterior segment at 8 weeks. Positive cells are seen in the anterior portion of the optic cup. (F) The ciliary body at 21 weeks. Positive staining is prominent in the epithelium (arrowheads). (G) The retina at 8 weeks. Positive cells are seen in the inner and outer neuroblastic layers. (H) The retina at 21 weeks. Positive cells are restricted to the ganglion cell layer and the inner and outer portions of the inner nuclear layer. $S=$ surface ectoderm; $L=$ lens; $O C=$ optic cup; $O S=$ optic stalk; GCL = ganglion cell layer; $I N L=$ inner nuclear layer ONL $=$ outer nuclear layer. $B a r=100 \mu m(A, C, D) ; 500 \mu m(B) ; 50 \mu m(E, F, G, H)$. 

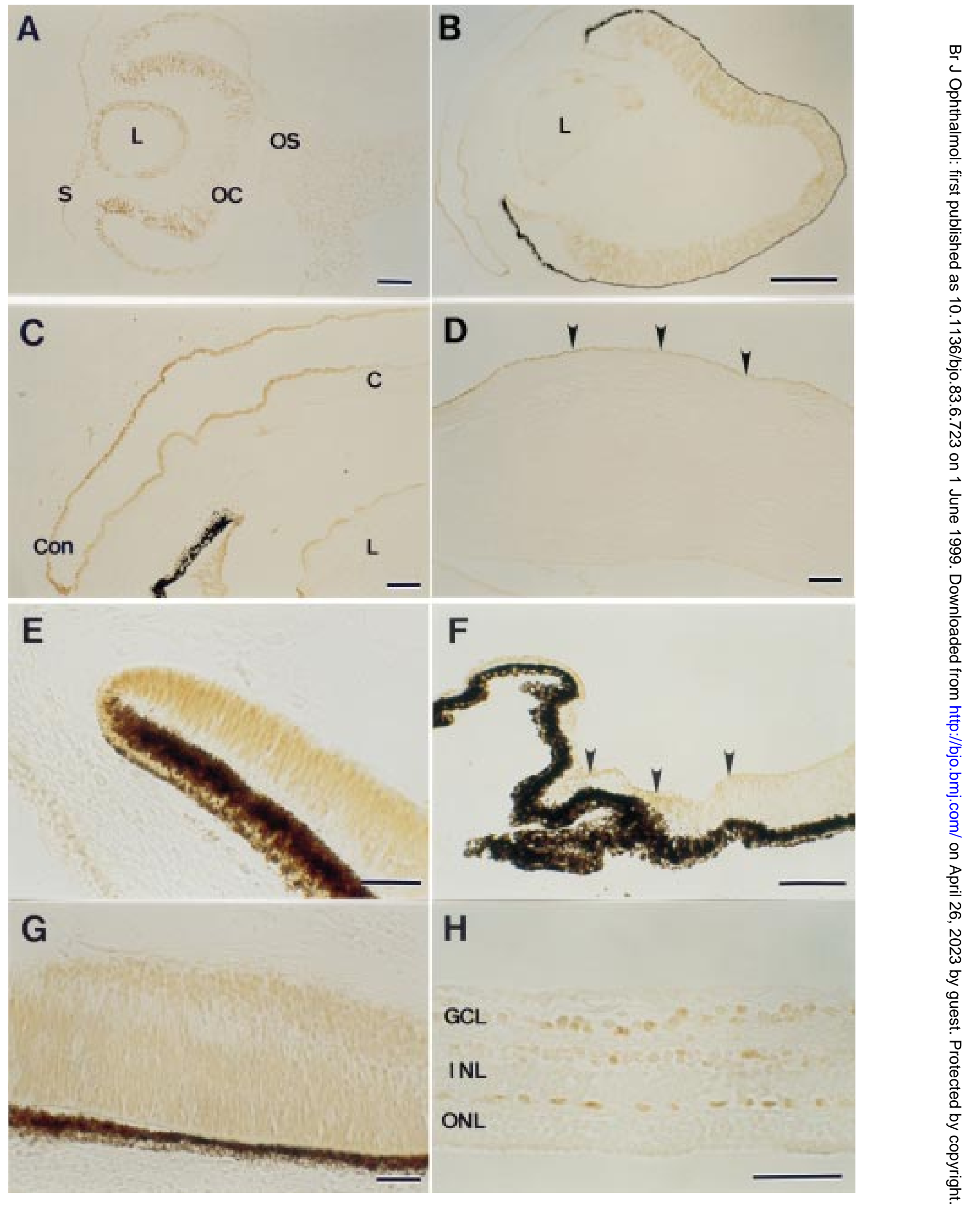
weeks; the mesenchymal cells were unstained (Fig 2A). Positive staining was observed from 8 to 10 weeks in the epithelia of the cornea, conjunctiva, and lens, and primordia of the iris and ciliary epithelia, and the inner and outer neuroblastic retinal layers, but vitreous vessels were unstained (Fig 2B, C, E). Positive staining persisted in the epithelia of the cornea, conjunctiva, lens, and ciliary body after 21 weeks, but tissue that differentiated from mesenchymal cells, including corneal stroma and endothelia, iris stroma, choroid, sclera, and vitreous vessels were unstained (Fig 2D, F). Retinal cells in all layers were positive for PAX6 at an early stage; however, at 21 and 22 weeks positive cells were restricted to the ganglion cell layer and the inner and outer portions of the inner nuclear layer, which probably differentiate amacrine and horizontal cells (Fig 2G, $\mathrm{H})$. The immunostaining procedure using alkaline phosphate and BCIP/NBT confirmed negative staining in cell nuclei of the retinal pigment epithelium (data not shown).

\section{Discussion}

As described in a previous study, ${ }^{9}$ western blot and immunohistochemical analysis showed that the PAX6 antibody detected Pax6 isoforms specifically but did not detect proteins of other Pax gene family members including Pax7 and Pax3. In this study, the PAX6 antibody cross reacted well with human ocular tissue because of the high conservation of the amino acid sequence between chicks and humans $(99.3 \%) .{ }^{19}$ Western blot analysis confirmed that the antibody specifically reacted to two isoforms of the human PAX6 with or without a 14 amino acid insertion encoded by exon $5 \mathrm{a}$ of a splice variant.

In the early stage, PAX6 was expressed in the tissue from the surface and neuroectoderms, including the corneal and conjunctival epithelia, lens, and optic cup, similar to immunohistochemical findings in murine ${ }^{5}$ and chick $^{9}$ eyes and in situ hybridisation in the 49 day old human eye. ${ }^{14}$ Adequate PAX6 expression in the ectoderms may be involved in determining position and subsequent inductive processes during early morphogenesis. In mutations of the small eye (Sey) locus, where the murine homologue of the PAX6 gene is located, the inactivation of both alleles results in the absence of eyes and severe craniofacial and central nervous system (CNS) disorders; inactivation of one allele results in the development of small eyes and mild CNS disorders. ${ }^{23}$ In humans, compound heterozygotes have no eyes and severe craniofacial and CNS disorders. ${ }^{16}$ Two missense mutations of the paired domain ${ }^{15}$ and proline-serine-threoninerich domain, ${ }^{24}$ a splice error, ${ }^{22}$ and a nonsense mutation have been found in anterior segment anomalies (summarised in the database at http://www.hgu.mrc.ac.uk/Softdata/PAX6). It is speculated that $P A X 6$ mutations disrupt the separation of the lens from the surface ectoderm and result in anterior segment malformations including Peters' anomaly. These abnormalities depend on the severity of the mutation in the PAX6.
In the development of the iris and ciliary body, PAX6 was first expressed at the rim of the optic cup, then in the non-pigmented ciliary epithelia in accordance with differentiation. PAX6 mutations may impair the signal for proper differentiation of these tissues and result in aniridia.

Peters' anomaly and aniridia also are associated with impaired migration of neural crest cells, which differentiate to form the trabecular tissue, the corneal stroma and endothelia, and the iris. ${ }^{25}$ However, we could not detect PAX6 expression in the mesenchymal cells. The small eye rat strain showed impaired migration of neural crest cells, although Pax6 transcripts were not observed in the neural crest derivatives by in situ hybridisation. ${ }^{26} \mathrm{~A}$ minute amount of PAX6 protein or mRNA in mesenchymal cells is undetectable by immunohistochemistry or in situ hybridisation, or PAX6 is absent in these cells. However, it is reasonable to consider that PAX6 may not be expressed in mesenchymal cells and not directly regulate their migration and differentiation. Pax6 binds the promoter of the N-CAM gene and activates its expression. ${ }^{10}$ Other factors, such as fibroblast growth factor, transforming growth factor $\beta$, and retinoic acid affect epithelialmesenchymal interactions. ${ }^{27}$ Although the relation between these factors and PAX6 is not clear, migration of neural crest cells possibly is induced by these factors and/or extracellular matrix and adhesion molecules from the surface ectoderm, lens epithelia, and the anterior portion of the optic cup.

PAX6 continued to be expressed in the epithelia of the cornea, conjunctiva, and lens at a late stage of development. In adult mice and monkeys it was expressed in epithelia of the cornea and conjunctiva. ${ }^{28}$ PAX6 may be expressed in these mitotically active and regenerating tissues through life, and abnormal expression may cause progressive corneal dystrophy. ${ }^{16}{ }^{17}$ The lens epithelia divide from 6 weeks' gestation and differentiate through development. PAX6 is one of the major regulators of the crystallin genes, ${ }^{11}$ thus inadequate expression may cause congenital cataract ${ }^{16}$ and be associated with aniridia and anterior segment anomalies.

In the retina, PAX6 was expressed in all cells of the optic cup, then in the inner and the outer neuroblastic layers at an early developmental stage. At 21 and 22 weeks, PAX6 was restricted to the ganglion cell layer and the inner and outer portions of the inner nuclear layer where amacrine and horizontal cells differentiate. These results are similar to the pattern of Pax6 expression in the developing chick retina. ${ }^{9}$ Restricted expression in ganglion and amacrine cells also was reported in the late phase of the developing Xenopus retina, ${ }^{7}$ and in the mature retina of mice, ${ }^{8}$ goldfish, ${ }^{6}$ and quail. ${ }^{29}$ During retinal development, multipotent precursor cells proliferate and migrate, they then differentiate to specific cell types, and cell death follows synaptogenesis. Histopathological study of human retina revealed that ganglion and Müller cells first differentiated, cone and amacrine cells then appeared, and 
rod and bipolar cells were distinguished during the late stage. ${ }^{30}$ Persistent PAX6 expression in the ganglion cells suggests a long period of maturation and its important role in retinal morphogenesis. The mature human retina does not have the ability and plasticity to regenerate neurons as in goldfish ${ }^{6}$; however, the developing retina has mitotically active cells that express PAX6. PAX6 dysfunction at a late stage causes associated or isolated foveal hypoplasia, ${ }^{18}$ because the fovea begins to develop at 30 weeks' gestation and development is complete at 4 months after birth. PAX6 later may be a determinant of postmitotic cell fate.

Mammalian homologues of the Drosophila so, eya, and dac genes, Six $3,{ }^{31}$ Eya $1-3,{ }^{32}$ and Dach, ${ }^{33}$ recently have been isolated. In the mouse, each gene is expressed in the various ocular tissue including the retina during development. In the retina, Chx10, another homeobox gene responsible for ocular retardation, was found to be restricted to bipolar and rod cells in mice ${ }^{34}$ and to bipolar cells in chicks. ${ }^{35}$ In the differentiating retina, Pax6 and Chx10 may specify cell types. There may be other homeobox genes, such as Prox $1,{ }^{34}$ Six $3,{ }^{31}$ and Eya $2,{ }^{32}$ that probably participate in retinal development. Thus, PAX6 may regulate the specific functions of these homeobox genes and dominate a regulatory hierarchy of differentiation, or PAX6 and others may play respective roles mosaically in determining individual cell fates during development. As yet, only one PAX6 mutation has been identified in a human retinal anomaly, isolated foveal hypoplasia ${ }^{18}$; however, further investigation may find mutations in other genes in more retinal anomalies.

Finally, we still have not clarified the distinct expression of PAX6 and PAX6-5a. The two DNA binding subdomains of the paired domain (N-terminal and C-terminal subdomains) negatively regulate their transactivation potentials for each other, and $5 \mathrm{a}$ insertion disrupts the autoregulatory mechanism and increases the transcriptional activity of C-terminal subdomain..$^{20} P A X 6$ gene dosage and appropriate levels of its transcriptional activity recently were shown to be important in ocular development. ${ }^{16}{ }^{36}$ PAX6-5a is supposed to affect the mechanism, regulate different target genes, and play distinct roles in determining cell fate and ocular morphogenesis. Further study of the specific expression of PAX6 and PAX6-5a may clarify multipotential roles in the morphogenesis of various ocular tissues through development.

1 Gehring WJ. The master control gene for morphogenesis and evolution at the eye. Genes Cells 1996;1:11-15.

2 Pignoni F, Hu B, Zavitz KH, et al. The eye-specification proteins So and Eya form a complex and regulate multiple steps in Drosophila eye development. Cell 1997;91:881-91.

3 Chen R, Amoui M, Zhang Z, et al. Dachshund and eyes absent proteins form a complex and function synergistically to induce ectopic eye development in Drosophila. Cell 1997;91:893-903.

4 Halder G, Callaerts P, Flister S, et al. Eyeless initiates the expression of both sine oculis and eyes absent during Drosophila compound eye development. Development 1998; 125:2181-91.

5 Walther C, Gruss P. Pax-6, a murine paired box gene, is expressed in the developing CNS. Development 1991;113: 1435-49.

6 Hitchcock PF, Macdonald RE, VanDeRyt JT, et al. Antibodies against PAX6 immunostain amacrine and ganglion cells and neuronal progenitors, but not rod precursors, in the normal and regenerating retina of the goldfish. 7 Neurobiol 1996:29:399-413.

7 Hirsch N, Harris WA. Xenopus Pax-6 and retinal development. F Neurobiol 1997;32:45-61

8 Davis JA, Reed RR. Role of Olf-1 and Pax-6 transcription factors in neurodevelopment. F Neurosci 1996;16:5082-94.

9 Kawakami A, Kimura-Kawakami M, Nomura T, et al. Distributions of PAX6 and PAX7 proteins suggest their involvement in both early and late phases at chick brain development. Mech Dev 1997;66:119-30.

10 Holst BD, Wang Y, Jones FS, et al. A binding site for Pax proteins regulates expression of the gene for the neural cell adhesion molecule in the embryonic spinal cord. Proc Natl Acad Sci USA 1997;94:1465-70.

11 Cvekl A, Piatigorsky J. Lens development and crystallin gene expression: many roles for Pax-6. BioEssays 1996;18: 621-30.

12 Stoykova A, Gotz M, Gruss P, et al. Pax6-dependent regulation of adhesive patterning, R-cadherin expression and boundary formation in developing forebrain. Development 1997;124:3765-77.

13 Sheng G, Thouvenot E, Schmucker D, et al. Direct regulation of rhodopsin 1 by Pax-6/eyeless in Drosophila: evidence for a conserved function in photoreceptors. Genes Dev 1997;11:1122-31.

14 Ton CCT, Hirvonen $\mathrm{H}$, Miwa $\mathrm{H}$, et al. Positional cloning and characterization of a paired box- and homeobox74

15 Hanson IM, Fletcher JM, Jordan T, et al. Mutations at the PAX6 locus are found in heterogeneous anterior segment malformations including Peters' anomaly. Nat Genet 1994; 6:168-73.

16 Glaser T, Jepeal L, Edwards JG, et al. PAX6 gene dosage effect in a family with congenital cataracts, aniridia, anophthalmia and central nervous system defects. Nat Genet 1994;7:463-71.

17 Mirzayans F, Pearce WG, MacDonald IM, et al. Mutation of the PAX6 gene in patients with autosomal dominant keratitis. Am F Hum Genet 1995;57:539-48.

18 Azuma N, Nishina S, Okuyama T, et al. PAX6 missense mutation in isolated foveal hypoplasia. Nat Genet 1996;13: $141-2$.

19 Gerard M, Abitbol M, Delezoide A-L, et al. PAX-genes expression during human embryonic development, a preliminary report. CR Acad Sci III 1995;318:57-66.

20 Yamaguchi Y, Sawada J, Yamada $M$, et al. Autoregulation of Pax6 transcriptional activation by two distinct DNAbinding subdomains of the paired domain. Genes Cells 1997;2:255-61.

21 Epstein J, Cai J, Glaser T, et al. Identification of a Pax paired domain recognition sequence and evidence for DNAdependent conformational changes. f Biol Chem 1994;269: 8355-61.

22 Epstein JA, Glaser T, Cai J, et al. Two independent and interactive DNA-binding subdomains of the Pax6 paired domain are regulated by alternative splicing. Genes Dev 1994;8:2022-34.

23 Hill RE, Favor J, Hogan BLM, et al. Mouse small eye results from mutation in a paired-like homeobox-containing gene. Nature 1991;354:522-5.

24 Azuma N, Yamada M. Missense mutation at the $\mathrm{C}$ terminus of the PAX6 gene in ocular anterior segmant anomalies. Invest Ophthalmol Vis Sci 1998;39:828-30.

25 Churchill A, Booth A. Genetics of aniridia and anterior segment dysgenesis. Br f Ophthalmol 1996;80:669-73.

26 Matsuo T, Osumi-Yamashita N, Noji S, et al. A mutation in the Pax-6 gene in rat small eye is associated with impaired the Pax-6 gene in rat small eye is associated with impaired
migration of midbrain crest cells. Nat Genet 1993;3:299migratio

27 Matsuo T. Molecular biological approaches to eye development. F Fpn Ophthalmol Soc 1993;97:1406-34

28 Koroma BM, Yang JM, Sundin OH. The Pax-6 homeobox gene is expressed throughout the corneal and conjunctival epithelia. Invest Ophthalmol Vis Sci 1997;30:108-20.

9 Martin P, Carriere C, Dozier C, et al. Characterization of a paired box- and homeobox-containing quail gene (PaxQNR) expressed in the neuroretina. Oncogene 1992;7: 1721-8.

30 Rhodes RH. A light microscopic study of the developing human neural retina. Am f Anat 1979;154:195-210.

31 Oliver G, Mailhos A, Wehr R, et al. Six3, a murine homologue of the sine oculis gene, demarcates the most anterior border of the developing neural plate and is expressed during eye development. Development 1995;121: expressed

32 Xu P-X, Woo I, Her H, et al. Mouse Eya homologues of the Drosophila eyes absent gene require Pax6 for expression in lens and nasal placode. Development 1997;124:219-31.

33 Hammond KL, Hanson IM, Brown AG, et al. Mammalian and Drosophila dachshund genes are related to the Ski proto-oncogene and are expressed in eye and limb. Mech Dev 1998;74:121-31.

34 Belecky-Adams T, Tomarev S, Li H-S, et al. Pax-6, Prox 1, and $\mathrm{Chx} 10$ homeobox gene expression correlates with phenotype fate of retinal precursor cells. Invest Ophthalmol Vis Sci 1997;38:1293-303.

35 Burmeister M, Novak J, Liang M-Y, et al. Ocular retardation mouse caused by Chx10 homeobox null allele: impaired retinal progenitor proliferation and bipolar cell differentiation. Nature Genet 1996;12:376-84.

36 Schedl A, Ross A, Lee M, et al. Influence of PAX6 gene dosage on development: overexpression causes severe eye abnormalities. Cell 1996;86:71-82. 